\title{
Nonuniversal gaugino masses in a magnetized toroidal compactification of SYM theories
}

\author{
Keigo Sumita \\ Department of Physics, Waseda University, \\ Tokyo 169-8555, Japan \\ E-mail: k.sumita@aoni.waseda.jp
}

ABSTRACT: This paper proposes a concrete model of nonuniversal gaugino masses on the basis of higher-dimensional supersymmetric Yang-Mills theories compactified on a magnetized factorizable torus, and we estimate the gauge coupling constants and gaugino masses in the model. In the magnetized toroidal compactifications, the four-dimensional effective action can be obtained analytically identifying its dependence on moduli fields, where the magnetic fluxes are able to yield the flavor structure of the minimal supersymmetric standard model (MSSM). The obtained gauge kinetic functions contains multi moduli fields and their dependence is nonuniversal for the three gauge fields. The nonuniversal gauge kinetic functions can lead to nonuniversal gaugino masses at a certain high energy scale (e.g. compactification scale). Our numerical analysis of them shows that, particular ratios of gaugino masses, which were found to enhance the Higgs boson mass and lead to "natural supersymmetry" in the MSSM, can be realized in our model, while the gauge couplings are unified as is achieved in the MSSM.

KEYwords: Supersymmetry Phenomenology, Phenomenology of Field Theories in Higher Dimensions

ARXIV EPRINT: 1507.04408 


\section{Contents}

1 Introduction $\quad 1$

2 Nonuniversal gaugino masses in the MSSM 3

3 Higher-dimensional SYM theories on magnetized tori 4

4 Superfield description of SYM systems $\quad 6$

5 A concrete model $\quad 8$

5.1 Pati-Salam models 9

$\begin{array}{ll}5.2 \text { A model of nonuniversal gaugino masses } & 10\end{array}$

$\begin{array}{lll}5.3 & \text { Gauge coupling unification and gaugino masses } & 14\end{array}$

$\begin{array}{lll}6 & \text { Conclusions and discussions } & 17\end{array}$

$\begin{array}{ll}\text { A RGE effects on the gauge coupling constants } & 18\end{array}$

\section{Introduction}

Supersymmetry (SUSY) has been regarded as one of the strong candidates for new physics beyond the standard model (SM), and the minimal supersymmetric standard model (MSSM) is quite supported by many people. Indeed, SUSY models has been proposed enormously so far and many of them respect main structure of the MSSM.

One of the great implications of SUSY is a cancellation of quantum corrections proportional to the square of cutoff scale. Low-energy SUSY ensures the stability of scalar masses, and the notorious fine-tunning problem for the stability of Higgs boson mass known in the SM is expected to be solved in the MSSM. From this point of view, we see that the low-energy SUSY is desirable. However, the mass of Higgs boson particle discovered at the Large Hadron Collider $[1,2]$ got the MSSM into a serious situation. The Higgs boson mass calculated at the tree-level of the MSSM is bounded by the $Z$ boson mass. A large amount of quantum corrections to the Higgs boson mass is required to realize the observed value. Dominant contributions of the quantum corrections is due to propagation of the SUSY particles. The Higgs boson mass is quite related to SUSY spectra in the MSSM or MSSM-like models. The sufficient amount of corrections requires a relatively heavy SUSY spectrum, but this is usually accompanied with another fine-tunning problem of the $\mu$-parameter (a SUSY higgsino mass parameter), that is, the so-called little hierarchy problem. As a result, a fine-tunning which is much harder than $\mathcal{O}(1 \%)$ cannot be avoided now in most of conventional parameter space of the MSSM. 
Another of the great impacts of SUSY in the MSSM is the gauge coupling unification. SUSY requires the presence of SUSY partners of the SM particles, and in the presence of such light particles charged under the SM gauge groups the renormalization group (RG) flows for the gauge coupling constants become different from those in the SM. As a result, it is well know that the three gauge couplings can be unified at a certain high energy scale within low-scale SUSY breaking scenarios. The unifying scale is about $M_{\mathrm{GUT}}=$ $2.0 \times 10^{16} \mathrm{GeV}$ in the MSSM.

In the unifications given by SUSY, we usually assume that the three gaugino masses are also unified at the same or near that scale. This popular assumption seems to be sensible, indeed, this has been adopted in many SUSY phenomenological studies. However, there is no reason for them to be constrained to unify, and we can freely choose their values at the $M_{\mathrm{GUT}}$ scale as input parameters in the MSSM. Considering a recent circumstances of particle physics experiments, that is, no detection of the SUSY particles and the relatively heavy mass of the Higgs boson, probably we are also required to investigate unconventional parameter space of the MSSM, i.e., nonuniversal gaugino masses.

The generic framework of nonuniversal gaugino masses were studied so far in the MSSM [3-5] and these works found an attractive feature. The gaugino masses are related to the Higgs boson mass in RG flows, and a certain range of the mass ratios will enhance the Higgs boson mass while a typical mass scale of SUSY spectra is not so much high. As the result, they found the fine tunning of $\mu$-parameter discussed above would be relaxed very well.

We consider unified theories behind the SM or ultraviolet (UV) complete theories in particle physics. In the top-down approaches of phenomenological studies on the basis of such theories, particle physics models are obtained as four-dimensional (4D) low-energy effective action. In such model building, the gaugino masses as well as the other masses and couplings cannot be set arbitrary, which should be determined by other structure of the theories, e.g., extra dimensional space. Although it is attractive that the natural parameter regions are still alive in the MSSM, we must remark how to realize such ratios of gaugino masses as a boundary condition at the $M_{\mathrm{GUT}}$ scale on the basis of such theories for a high energy physics. This paper provides a realization of nonuniversal gaugino masses on the basis of higher-dimensional SYM theories which appear in low-energy limits of superstring theories.

In model building based on higher-dimensional SYM theories, structure of extra dimensions of space is the most significant issue to obtain a realistic model with $4 \mathrm{D}$ chiral spectra like the SM. It is known that toroidal compactifications with magnetic fluxes are able to yield such a spectrum in higher-dimensional SYM theories [6, 7]. The magnetized toroidal compactification have been actively studied, and some concrete models have been constructed, where the flavor structure of the SM, such as, the three generations of matters and their hierarchical spectrum, are obtained. In particular, ref. [8, 9] proposed such a model based on a 10D U(8) SYM theories, and the mass spectrum of the SUSY particles as well as the SM particles were studied to verify this model. These works owe mainly their model building to an $\mathcal{N}=1$ superfield description of ten-dimensional (10D) magnetized SYM theories given in ref. [10]. That proposed a systematic way of dimensional reduction of the magnetized SYM theories with the superfield description, and derived the $4 \mathrm{D}$ effective 
action identifying its dependence on a dilaton and moduli superfields. In the superspace formulation, an $\mathcal{N}=1$ SUSY out of the full $\mathcal{N}=4$ SUSY (counted by the 4 D supercharges) of 10D SYM theories is described manifestly, and $\mathcal{N}=1$ SUSY configurations of magnetic fluxes is facilitated to study.

The superfield description for 10D SYM theories have been recently extended to be able to apply to $(4+2 \mathrm{n})$-dimensional SYM theories and their mixtures which are well motivated by D-brane pictures of superstring theories [11]. This extension allows a large variety of model building, and especially, we find that the mixture is a suitable foundation to construct a model of nonuniversal gaugino masses. This paper shows such a concrete model based on a mixture of a six-dimensional (6D) SYM theory and a 10D SYM theory compactified on magnetized tori, and estimates the three SM gauge coupling constants and the gaugino masses.

This paper is constructed as follows. We briefly review the nonuniversal gaugino masses in the MSSM in section 2. Section 3 gives an overview of higher-dimensional SYM theories compactified on a factorizable torus with magnetic fluxes. This section shows how to realize the flavor structure of the SM by magnetic fluxes. In section4, the superfield description of the magnetized SYM theories is briefly reviewed. The way of dimensional reduction and identifying the moduli dependence will become clear. Section 5 is devoted to show our results. A concrete model will be proposed, where we study the gaugino masses to verify that our model accommodate a realization of the nonuniversal gaugino masses desired from the phenomenological point of view. Section 6 contains conclusions and discussions.

\section{Nonuniversal gaugino masses in the MSSM}

We shortly review nonuniversal gaugino masses in the MSSM, which shows one of our motivations of this work. In a popular assumption, three gaugino masses are set to be unified at the $M_{\mathrm{GUT}}$ scale for simplicity,

$$
M_{1}\left(M_{\mathrm{GUT}}\right)=M_{2}\left(M_{\mathrm{GUT}}\right)=M_{3}\left(M_{\mathrm{GUT}}\right),
$$

where $M_{i}(i=1,2,3)$ are the gaugino mass parameters of $\mathrm{U}(1)_{Y}, \mathrm{SU}(2)_{L}$ and $\mathrm{SU}(3)_{C}$, respectively. However, they are just free parameters and nothing restricts their values to be degenerate in the MSSM. It is also possible to consider nonuniversal gaugino masses in the MSSM.

The nonuniversal gaugino masses in the MSSM were studied in refs. [3-5], where they found that nonuniversal gaugino masses with certain ratios enhance the Higgs boson mass through the RG effects, and the fine-tunning of so-called $\mu$-parameter will be relaxed. To discuss more details, we define the degree of fine-tunning as $100 / \Delta_{\mu}(\%)[12]$ with

$$
\Delta_{\mu}=\left|\frac{\partial \log m_{Z}^{2}}{\partial \log \mu^{2}}\right| \text {. }
$$

In ref. [4], the authors studied impact of the nonuniversal gaugino masses on the Higgs boson mass and the SUSY spectrum in the light of the LHC data with a simple ansatz for other parameters, e.g., Yukawa couplings. As the result, in the range of

$$
3.0 \lesssim \frac{M_{2}\left(M_{\mathrm{GUT}}\right)}{M_{3}\left(M_{\mathrm{GUT}}\right)} \lesssim 5.5, \quad-3.0 \lesssim \frac{M_{1}\left(M_{\mathrm{GUT}}\right)}{M_{3}\left(M_{\mathrm{GUT}}\right)},
$$


the $126 \mathrm{GeV}$ Higgs boson will be realized with the tunning of $\mathcal{O}(1)(\%)$. Furthermore, in the following region,

$$
5.2 \lesssim \frac{M_{2}\left(M_{\mathrm{GUT}}\right)}{M_{3}\left(M_{\mathrm{GUT}}\right)} \lesssim 5.5
$$

the fine-tunning of $\mu$-parameter will be relaxed as well as $\mathcal{O}(10)(\%)$.

In the generic framework of SUSY models, we require the presence of hidden sector sequestered from the MSSM sector, where SUSY should be spontaneously broken. The SUSY breaking contribution is mediated to the MSSM sector, and thus, the mediation mechanism determines the SUSY spectrum as a boundary condition of RG flows at a certain high energy scale.

Three mediation mechanisms due to moduli fields, conformal anomaly [13, 14] and gauge interactions [15-17] are famous and available in generic frameworks. These mediation mechanisms and their combinations have been actively studied so far, and we know their phenomenological features precisely. According to that, it is impossible to realize the the desirable ratio $M_{2} / M_{3} \sim 5$ within the anomaly and gauge mediation mechanisms, and we see that the SUSY breaking contributions mediated by moduli fields through nonuniversal gauge kinetic functions are indispensable to obtain the ideal ones.

When the gauge kinetic functions of $\mathrm{SU}(3)_{C}, \mathrm{SU}(2)_{L}$ and $\mathrm{U}(1)_{Y}$ can be flexibly chosen differently from each other in terms of its dependence on moduli fields, the gaugino masses are controllable. However, in models based on the unified theories, the couplings of moduli fields to the MSSM fields are determined by structure of extra dimensional space. We give a realization of nonuniversal gaugino masses on the basis of a concrete model derived from a higher-dimensional SYM system compactified on magnetized tori.

\section{Higher-dimensional SYM theories on magnetized tori}

This section reviews higher-dimensional SYM theories compactified on two-dimensional (2D) tori with magnetic fluxes. Higher-dimensional fields are expanded into zero-modes and multiple Kaluza-Klein (KK) modes. In this section, we derive 4D effective actions from the magnetized SYM theories focusing on the zero-modes. KK modes are considered to be heavy enough to be decoupled in the low-energy effective field theories. To show typical features of magnetized toroidal compactifications, we concentrate on a two-dimensional torus whose coordinates are denoted by $(x, y)$. The line element is given by

$$
d s^{2}=g_{i j} d x^{i} d x^{j},
$$

where the metric $g$ is

$$
g=(2 \pi R)^{2}\left(\begin{array}{cc}
1 & \operatorname{Re} \tau \\
\operatorname{Re} \tau & |\tau|^{2}
\end{array}\right) .
$$

Parameters $\tau$ and $R$ determine the shape and size of this torus.

We consider a 2D spinor field on this torus,

$$
\psi=\left(\begin{array}{l}
\psi_{+} \\
\psi_{-}
\end{array}\right) .
$$


The zero-mode equations for these fields are given by the Dirac operator on this internal space as

$$
\begin{aligned}
& \bar{\partial}_{z} \psi_{+}+\left[\bar{A}_{z}, \psi_{+}\right]=0 \\
& \partial_{z} \psi_{-}-\left[A_{z}, \psi_{-}\right]=0,
\end{aligned}
$$

where we use complex coordinates $z$ and $2 \mathrm{D}$ complex vector $A_{z}$ defined by two real coordinates $x, y$ of this torus and two components of vector fields $\left(A_{x}, A_{y}\right)$ as follows,

$$
\begin{aligned}
z & \equiv \frac{1}{2}(x+\tau y) \\
A_{z} & \equiv-\frac{1}{\operatorname{Im} \tau}\left(\tau^{*} A_{x}-A_{y}\right) .
\end{aligned}
$$

Toroidal periodicity for the two coordinates is expressed by $z \sim z+1$ and $z \sim z+\tau$.

Considering a nontrivial configuration of gauge potential, we introduce magnetic fluxes on this torus, and the configuration is

$$
A_{z}=\frac{\pi}{\operatorname{Im} \tau}(M \bar{z}+\bar{\zeta})
$$

where the magnetic flux is given by an $N \times N$ matrix $M$ in $\mathrm{U}(N)$ theories. A continuous Wilson lines can also be introduced, which is identified as a constant term of gauge potential and denoted by an $N \times N$ matrix $\zeta$ here. This paper considers only simple Abelian forms of the flux and Wilson line because those are sufficient and necessary to construct particle physics models, thus offdiagonal entries of $M$ and $\zeta$ are set to be vanishing. Note that the nonvanishing entries of $M$ must be integer values because of the Dirac's quantization condition. In the case of $N=2$, if the two diagonal elements of flux matrix take different values from each other, $\mathrm{U}(2)$ gauge symmetry is broken down to $\mathrm{U}(1) \times \mathrm{U}(1)$. Thus, in generic $\mathrm{U}(N)$ theories, the magnetic fluxes $M$ can lead to gauge symmetry breaking as $\mathrm{U}(N) \rightarrow$ $\prod_{a} \mathrm{U}\left(N_{a}\right)\left(\mathrm{U}\left(N_{a}\right)\right.$ is a remaining gauge subgroup of $\left.\mathrm{U}(N)\right)$. This Wilson lines $\zeta$ are also able to induce such a gauge symmetry breaking in the same way as the magnetic fluxes.

In the zero-mode equation (3.1) for $\psi_{+}$, we can elicit a bifundamental representation $\left(N_{a}, \bar{N}_{b}\right)$ of $\mathrm{U}\left(N_{a}\right) \times \mathrm{U}\left(N_{b}\right)$ as

$$
\left[\bar{\partial}_{z}+\frac{\pi}{2 \operatorname{Im} \tau}\left(M_{a b} z+\zeta_{a b}\right)\right]\left(\psi_{+}\right)_{a b}=0,
$$

where a bifundamental representation $\left(N_{a}, \bar{N}_{b}\right)$ contained in $\psi_{+}$is denoted by $\left(\psi_{+}\right)_{a b}$. A magnetic flux felt by it is defined as $M_{a b} \equiv M_{a}-M_{b}$, and a Wilson line is also defined as $\zeta_{a b} \equiv \zeta_{a}-\zeta_{b}$. According to ref. [7], this equation has $M_{a b}$ normalizable solutions when $M_{a b}>0$, and then, zero-modes of the same representation $\left(N_{a}, \bar{N}_{b}\right)$ contained in the other spinor $\psi_{-}$are eliminated because a relative sign of the zero-mode equation (3.2) is different. On the contrary, when $M_{a b}<0,\left(\psi_{-}\right)_{a b}$ has $\left|M_{a b}\right|$ well-defined zero-modes and the other $\left(\psi_{+}\right)_{a b}$ has none. Thus, the magnetic fluxes cause a kind of chirality projection which is to generate a $4 \mathrm{D}$ chiral spectrum like the SM.

The degenerate zero-modes appear corresponding to the magnitude of fluxes, which we can identify with the generations of the SM. Their wavefunctions can be obtained analytically, expressed by using the Jacobi-theta functions. Moreover, they have a Gaussian 
profile on the torus, and their localized points on the magnetized torus are different from each other. Since overlap integrals of the zero-mode wavefunctions determine the magnitude of their $4 \mathrm{D}$ effective couplings, zero-modes localized far away from each other yield a suppressed coupling, which can give a hierarchical structure to Yukawa couplings. It is also attractive that the integrals of zero-mode wavefunctions on the magnetized torus can be performed analytically. The simple expression for Yukawa couplings [7] and higher-order couplings [18] were obtained. Summarizing the above, the magnetic fluxes are able to yield the flavor structure of the SM, such as, the three generations and their hierarchical masses and mixing angles.

\section{Superfield description of SYM systems}

This section introduces a superfield description of higher-dimensional SYM theories on the magnetized tori [10], which is extremely useful, especially for constructing a particle physics model with an $\mathcal{N}=1$ SUSY vacuum configuration. A $4 \mathrm{D}$ effective action with $\mathcal{N}=1$ SUSY can be derived from magnetized SYM theories in the superfield description, identifying its dependence on the moduli fields.

Higher-dimensional field theories have higher-dimensional SUSY as $\mathcal{N}=2,3$ and 4 counted by the $4 \mathrm{D}$ supercharges, but these theories can be described in the $4 \mathrm{D} \mathcal{N}=1$ superspace formulation focusing on an $\mathcal{N}=1$ SUSY of the whole higher-dimensional SUSY in the following way. First, we consider a 10D SYM action compactified on three 2D tori,

$$
S=\int d^{10} X \sqrt{-G}\left\{-\frac{1}{4 g^{2}} \operatorname{Tr}\left(F^{M N} F_{M N}\right)+\frac{i}{2 g^{2}}\left(\bar{\lambda} \Gamma^{M} D_{M} \lambda\right)\right\},
$$

where $g$ is the gauge coupling constant. Capital Latins $M, N$ run for the $10 \mathrm{D}$ spacetime coordinate, and the 10D field strength $F^{M N}$, covariant derivative $D_{M}$ and gamma matrix $\Gamma^{M}$ are contracted by the $10 \mathrm{D}$ metric $G_{M N}$. The $10 \mathrm{D}$ line element is

$$
d s^{2}=\eta_{\mu \nu} d x^{\mu} d x^{\nu}+g_{m n} d x^{m} d x^{n}
$$

where $\mu, \nu: 0,1,2,3$ and $m, n: 4,5, \ldots, 9$. The $4 \mathrm{D}$ Minkowski spacetime is given by $\eta=\operatorname{diag}(-,+,+,+)$ and $g_{m n}$ gives three tori, $T^{2} \times T^{2} \times T^{2}$.

Field contents of this theory is given by a $10 \mathrm{D}$ vector field $A_{M}$ and a Majorana-Weyl spinor field $\lambda$, satisfying conditions $\Gamma^{10} \lambda=+\lambda$ and $\lambda^{C}=\lambda\left(\Gamma^{10}\right.$ is a 10D chirality operator and $\mathrm{C}$ represents the charge conjugate. ). To make an $\mathcal{N}=1$ SUSY manifest, the 10D vector field is decomposed into a $4 \mathrm{D}$ vector field and three complex fields as

$$
A_{\mu}, \quad A_{i} \equiv-\frac{1}{\operatorname{Im} \tau_{i}}\left(\tau_{i}^{*} A_{m}-A_{n}\right),
$$

where $i=1,2,3$ and $(m, n)=(2+2 i, 3+2 i)$. The 10D spinor field is also decomposed into the following four $4 \mathrm{D}$ Weyl spinor fields,

$$
\lambda_{0} \equiv \lambda_{+++}, \quad \lambda_{1} \equiv \lambda_{+--}, \quad \lambda_{2} \equiv \lambda_{-+-}, \quad \lambda_{3} \equiv \lambda_{--+},
$$


where signs \pm represent a chirality on each torus, e.g., $\lambda_{+--}$has a positive chirality on the first torus and negative ones on the other tori. 4D Spinor fields with the other chirality are not contained in the 10D Majorana-Weyl spinor because of $\Gamma^{10} \lambda=+\lambda$.

The component fields form $4 \mathrm{D} \mathcal{N}=1$ vector multiplets and chiral multiplets,

$$
\left\{A \mu, \lambda_{0}\right\}, \quad\left\{A_{i}, \lambda_{i}\right\}
$$

These supermultiplets are embedded into a vector superfield and three chiral superfields as follows,

$$
\begin{aligned}
V & \equiv-\theta \sigma^{\mu} \bar{\theta} A_{\mu}+i \bar{\theta} \bar{\theta} \theta \lambda_{0}-i \theta \theta \bar{\theta} \bar{\lambda}_{0}+\frac{1}{2} \theta \theta \bar{\theta} \bar{\theta} D \\
\phi_{i} & \equiv \frac{1}{\sqrt{2}} A_{i}+\sqrt{2} \theta \lambda_{i}+\theta \theta F_{i}
\end{aligned}
$$

where two-component spinors $\theta$ and $\bar{\theta}$ are $4 \mathrm{D} \mathcal{N}=1$ supercoordinates.

The 10D SYM action (4.1) is expressed in the $4 \mathrm{D} \mathcal{N}=1$ superspace formulation with the above superfields as [19, 20],

$$
S=\int d^{10} X \sqrt{-G}\left[\int d^{4} \theta \mathcal{K}+\left\{\int d^{2} \theta\left(\frac{1}{4 g^{2}} \mathcal{W}^{\alpha} \mathcal{W}_{\alpha}+\mathcal{W}\right)+\text { h.c. }\right\}\right]
$$

where three functions $\mathcal{K}, \mathcal{W}$ and $\mathcal{W}^{\alpha}$ are given by

$$
\begin{aligned}
\mathcal{K} & =\frac{2}{g^{2}} h^{\bar{i} j} \operatorname{Tr}\left[\left(\sqrt{2} \bar{\partial}_{\bar{i}}+\bar{\phi}_{\bar{i}}\right) e^{-V}\left(-\sqrt{2} \partial_{j}+\phi_{j}\right) e^{V}+\bar{\partial}_{\bar{i}} e^{-V} \partial_{j} e^{V}\right]+\mathcal{K}_{\mathrm{WZW}}, \\
\mathcal{W} & =\frac{1}{g^{2}} \epsilon^{\mathrm{ijk}} e_{\mathrm{i}}{ }^{i} e_{\mathrm{j}}^{j} e_{\mathrm{k}}^{k} \operatorname{Tr}\left[\sqrt{2} \phi_{i}\left(\partial_{j} \phi_{k}-\frac{1}{3 \sqrt{2}}\left[\phi_{j}, \phi_{k}\right]\right)\right] \\
\mathcal{W}_{\alpha} & =-\frac{1}{4} \bar{D} \bar{D} e^{-V} D_{\alpha} e^{V}
\end{aligned}
$$

In these expressions, $h^{\bar{i} j}$ and $e_{\mathrm{i}}{ }^{i}$ express the metric and fielbein of each torus. The supercovariant derivatives are denoted by $D_{\alpha}$ and $\bar{D}_{\dot{\alpha}}$.

The field equations for auxiliary fields $D$ and $F_{i}$ are given by

$$
\begin{aligned}
& D=-h^{\bar{i} j}\left(\bar{\partial}_{\bar{i}} A_{j}+\partial_{j} \bar{A}_{\bar{i}}+\frac{1}{2}\left[\bar{A}_{\bar{i}}, A_{j}\right]\right), \\
& \bar{F}_{\bar{i}}=-h_{j i} \bar{i}^{\mathrm{jkl}} e_{\mathrm{j}}^{j} e_{\mathrm{k}}{ }^{k} e_{L}^{l}\left(\partial_{k} A_{l}-\frac{1}{4}\left[A_{k}, A_{l}\right]\right) .
\end{aligned}
$$

The $\mathcal{N}=1$ SUSY expressed by the superfield formulation is preserved as long as the Vacuum Expectation Values (VEVs) of these auxiliary fields are vanishing.

The magnetic fluxes are introduced as

$$
\left\langle A_{i}\right\rangle=\frac{\pi}{\operatorname{Im} \tau_{i}}\left(M^{(i)} \bar{z}_{i}+\bar{\zeta}^{(i)}\right) .
$$


These magnetic fluxes should satisfy a condition $\langle D\rangle=\left\langle F_{i}\right\rangle=0$ to preserve the $\mathcal{N}=1$ SUSY. On this magnetized background, the zero-mode equations for the superfield $\phi_{j}$ on the $i$-th torus are obtained as

$$
\begin{aligned}
& {\left[\bar{\partial}_{\bar{i}}+\frac{\pi}{2 \operatorname{Im} \tau_{i}}\left(M_{a b}^{(i)} z_{i}+\zeta_{a b}^{(i)}\right)\right]\left(f_{j}^{(i)}\right)_{a b}=0 \quad \text { for } \quad i=j,} \\
& {\left[\partial_{i}-\frac{\pi}{2 \operatorname{Im} \tau_{i}}\left(M_{a b}^{(i)} \bar{z}_{\bar{i}}+\bar{\zeta}_{a b}^{(i)}\right)\right]\left(f_{j}^{(i)}\right)_{a b}=0 \quad \text { for } \quad i \neq j,}
\end{aligned}
$$

where $\left(f_{j}^{(i)}\right)_{a b}$ represents a zero-mode wavefunction on the $i$-th torus of a bifundamental representation $\left(N_{a}, \bar{N}_{b}\right)$ contained in $\phi_{j}$. These equations for $i=j$ and $i \neq j$ have the same form as the Dirac equations (3.1) and (3.2), respectively. Thus, their zero-mode wavefunctions are obtained and the $4 \mathrm{D}$ effective action can also be derived analytically in this superfield description. The gauge fields of the MSSM are accommodated by diagonal parts of the vector superfield $V$ which do not feel the Abelian magnetic fluxes. Offdiagonal component of $V$ get heavy because of the partial gauge symmetry breaking, and they will be decoupled.

The $4 \mathrm{D}$ effective action generically contains some parameters. such as, the gauge coupling constant, torus radius and complex structure. These parameters are promoted to moduli fields in supergravity framework. It is known that a conventional relation between the VEVs of moduli fields and the parameters as follows [21],

$$
\operatorname{Re}\langle S\rangle=e^{-\langle\phi\rangle} \alpha^{\prime-3} \mathcal{A}^{(i)}, \quad \operatorname{Re}\left\langle T_{r}\right\rangle=e^{-\langle\phi\rangle} \alpha^{\prime-1} \mathcal{A}^{(r)}, \quad \operatorname{Re}\left\langle U_{r}\right\rangle=i \bar{\tau}_{r},
$$

where $\mathcal{A}^{(r)}$ represents the area of the $r$-th torus and the 10D dilaton field determines the 10D gauge coupling,

$$
g=e^{\langle\phi\rangle / 2} \alpha^{\prime 3 / 2}
$$

This relation allows us to determine the moduli dependence of the $4 \mathrm{D}$ effective actions. Thus, explicit forms of gauge kinetic functions, Kähler metrics and holomorphic Yukawa couplings as functions of the moduli fields are obtained.

This systematic procedure to derive $4 \mathrm{D}$ effective supergravity actions from 10D SYM theories compactified on magnetized tori was extended to apply to $(4+2 n)$-dimensional SYM theories and their mixtures in ref. [11]. In that paper, two specific $4 \mathrm{D}$ effective supergravity actions with the explicit moduli dependence were shown to demonstrate the extended procedure. They were derived from two SYM systems well motivated by stable Dbrane systems. One is a mixture of a 4D SYM theory and an eight-dimensional SYM theory. The other is composed of a 6D SYM theory and a 10D SYM theory. In this paper, we consider the latter SYM system to realize the nonuniversal gaugino masses (Note the two systems are essentially equivalent because they should be related to each other by T-duality).

\section{A concrete model}

We construct an MSSM-like model based on a mixture of a 10D SYM theory compactified on three tori $\left(T^{2}\right)_{1} \times\left(T^{2}\right)_{2} \times\left(T^{2}\right)_{3}$ and a 6 D SYM theory on $\left(T^{2}\right)_{1}$. Although the D-brane physics is one of our motivations to consider this SYM system, we are just starting from the SYM theories here and will not mention whole consistency for string models in this paper. 


\begin{tabular}{|cccc|}
\hline Representations & MSSM fields & Fluxes on $\left(T^{2}\right)_{1}$ & \# of gen. \\
\hline$(4, \overline{2}, 1)$ & Left-handed & $m_{C}^{(1)}-m_{L}^{(1)}=-3$ & 3 \\
$(\overline{4}, 1,2)$ & Right-handed & $m_{R}^{(1)}-m_{C}^{(1)}=-3$ & 3 \\
$(1,2, \overline{2})$ & Higgs & $m_{L}^{(1)}-m_{R}^{(1)}=+6$ & 6 \\
\hline
\end{tabular}

Table 1. Field contents and magnetic fluxes felt by them on the first torus in the Pati-Salam model.

\subsection{Pati-Salam models}

One of the most important things in such model buildings in the magnetized toroidal compactifications is that all the flavor structure must originate from a single torus. For example, if the three generations of left-handed quarks are generated by magnetic fluxes on the first torus and those of right-handed quarks are induced on the second torus, rank of their Yukawa matrix is reduced to one, and two of the three generations will remain massless after the electroweak symmetry breaking. Thus, to construct a three-generation model in the SYM system given in the top of this section, the magnetic fluxes on the first torus $\left(T^{2}\right)_{1}$ must yield whole the flavor structure of the MSSM, and the magnetic fluxes on the other tori should be determined not to disturb the structure and to satisfy conditions to preserve the $\mathcal{N}=1$ SUSY.

A unique configuration of magnetic fluxes to generate whole the flavor structure of SM on a single torus was found $[10]^{1}$ and an MSSM-like model was proposed starting from a 10D U(8) SYM theory. The U(8) gauge group was broken down to the PatiSalam gauge group, $\mathrm{U}(4)_{C} \times \mathrm{U}(2)_{L} \times \mathrm{U}(2)_{R}$, which is to lead to a SM-like gauge group $\mathrm{SU}(3)_{C} \times \mathrm{SU}(2)_{L} \times(\mathrm{U}(1))^{5}$ by introducing Wilson lines. To give an overview of the model, we define the following matrix $M^{(i)}$ which appears in the VEV of eq. (4.4),

$$
M^{(i)}=\left(\begin{array}{ccc}
m_{C}^{(i)} \times \mathbf{1}_{4} & 0 & 0 \\
0 & m_{L}^{(i)} \times \mathbf{1}_{2} & 0 \\
0 & 0 & m_{R}^{(i)} \times \mathbf{1}_{2}
\end{array}\right)
$$

The suitable configuration is then shown as

$$
\left(m_{C}^{(1)}, m_{L}^{(1)}, m_{R}^{(1)}\right)=(0,+3,-3)
$$

In the Pati-Salam group, $(4, \overline{2}, 1)$ representation contains the left-handed matter fields and $(\overline{4}, 1,2)$ representation does the right-handed matters. Higgs multiplets are carried by $(1,2, \overline{2})$ representation, and each representations feel the magnetic fluxes of $m_{C}^{(i)}-m_{L}^{(i)}$, $m_{R}^{(i)}-m_{C}^{(i)}$ and $m_{L}^{(i)}-m_{R}^{(i)}$, respectively. We summarize them in table 1 . On the first torus, these magnetic fluxes induce three-generations of left- and right-handed matters and six-generations of Higgs fields. The presence of these multiple Higgs fields is a generic feature of the magnetized SYM theory as well as D-brane models, and we identify a linearcombinations of the six ones with the MSSM Higgs multiplet.

\footnotetext{
${ }^{1}$ Orbifold projections lead to other flux configurations to construct three-generation models [22-24].
} 
In the single 10D SYM model, flux configurations on the other two tori were determined as

$$
\begin{aligned}
& \left(m_{C}^{(2)}, m_{L}^{(2)}, m_{R}^{(2)}\right)=(0,-1,0) \\
& \left(m_{C}^{(3)}, m_{L}^{(3)}, m_{R}^{(3)}\right)=(0,0,+1),
\end{aligned}
$$

which will not spoil the flavor structure generated on the first torus and satisfy the SUSY preserving condition $\langle D\rangle=\left\langle F_{i}\right\rangle=0$ with the following relation of the torus area,

$$
\mathcal{A}^{(1)} / \mathcal{A}^{(2)}=\mathcal{A}^{(1)} / \mathcal{A}^{(3)}=3 .
$$

We respect the Pati-Salam gauge and this configuration of magnetic fluxes also in this paper, and those can be embedded into a mixture of 10D U $(N)$ SYM theory compactified on three tori and $6 \mathrm{D} \mathrm{U}(8-N)$ theory on $\left(T^{2}\right)_{1}$. Thus, we first divide the Pati-Salam group into two parts. Table 1 reads that the Higgs multiplets contained in representation $(1,2, \overline{2})$ feel positive magnetic fluxes, +6 , on the first torus. This means that the Higgs fields originate from $\phi_{1}$ which is a superfield consisting of $A_{1}$ and $\lambda_{1}$, because the other chiral superfields require negative magnetic fluxes to have zero-modes on the first torus. In the action of 10D $\mathrm{U}(N)$ and $6 \mathrm{D} \mathrm{U}(8-N)$ SYM theories described in the superspace formulation, all bifundamental representations of $\mathrm{U}(N) \times \mathrm{U}(8-N)$ are completely eliminated in $\phi_{1}$. This is because the bifundamental representations are to form a hypermultiplet under the $6 \mathrm{D}$ $(4 \mathrm{D} \mathcal{N}=2)$ SUSY but the chiral superfield $\phi_{1}$ is consists of vector components of the $6 \mathrm{D}$ theory. Therefore, the Higgs multiplets, $(1,2, \overline{2})$ representations of the Pati-Salam gauge group, must be contained in adjoint representations of $\mathrm{U}(N)$ or $\mathrm{U}(8-N)$. We can determine from this discussion that the Pati-Salam model is embedded into the SYM system, being divided into $\mathrm{U}(4)_{C}$ and $\mathrm{U}(4)_{L R} \supset \mathrm{U}(2)_{L} \times \mathrm{U}(2)_{R}$.

With this division of the Pati-Salam gauge group, two types of SYM systems are available: one consists of the $10 \mathrm{D} \mathrm{U}(4)_{C}$ SYM and the 6D U(4) ${ }_{L R}$ SYM theories, and the other of the $10 \mathrm{D} \mathrm{U}(4)_{L R} \mathrm{SYM}$ and the $6 \mathrm{D} \mathrm{U}(4)_{C}$ SYM theories. In the former case, $\mathrm{U}(4)_{L R}$ has to be broken down to $\mathrm{U}(2)_{L} \times \mathrm{U}(2)_{R}$ by the magnetic fluxes on the first torus, which fluxes must also break the $\mathcal{N}=1$ SUSY because there is no other contribution of magnetic fluxes in the $\mathrm{U}(4)_{L R}$ sector to satisfy $\langle D\rangle=0$. On the contrary, magnetic fluxes of the $\mathrm{U}(4)_{L R}$ sector are given on three tori in the other case. Therefore, only the latter case can take SUSY preserving configurations. We have found the suitable SYM system, which is studied in the next subsection.

\subsection{A model of nonuniversal gaugino masses}

We consider the SYM system consisting of the $10 \mathrm{D} \mathrm{U}(4)_{L R} \mathrm{SYM}$ and the $6 \mathrm{D} \mathrm{U}(4)_{C} \mathrm{SYM}$ theories, which are compactified on magnetized $\left(T^{2}\right)_{1} \times\left(T^{2}\right)_{2} \times\left(T^{2}\right)_{3}$ and $\left(T^{2}\right)_{1}$, respectively. The configuration of magnetic fluxes is parametrized as

$$
M_{C}^{(1)}=m_{C} \times \mathbf{1}_{4},
$$

and

$$
M_{L R}^{(i)}=\left(\begin{array}{cc}
m_{L}^{(i)} \times \mathbf{1}_{2} & 0 \\
0 & m_{R}^{(i)} \times \mathbf{1}_{2}
\end{array}\right)
$$


where $M_{C}^{(1)}$ is the magnetic flux in the $\mathrm{U}(4)_{C}$ SYM theory, and $M_{L R}^{(i)}(i=1,2,3)$ are those in $\mathrm{U}(4)_{L R} \mathrm{SYM}$ theory, on each torus. These parameters are set as

$$
\begin{aligned}
m_{C} & =0 \\
\left(m_{L}^{(1)}, m_{R}^{(1)}\right) & =(+3,-3) \\
\left(m_{L}^{(2)}, m_{R}^{(2)}\right) & =(-1,0) \\
\left(m_{L}^{(3)}, m_{R}^{(3)}\right) & =(0,+1) .
\end{aligned}
$$

The magnetic fluxes on the first torus $M_{C}^{(1)}$ and $M_{L R}^{(1)}$ yield the same flavors as is shown in table 1 . The others are determined to preserve the $\mathcal{N}=1$ SUSY without changes of the flavor structure. The vanishing $m_{C}$ is also a key to preserve the SUSY.

Although there remain a few massless adjoint fields other than the MSSM fields in the $4 \mathrm{D}$ effective theory, all chiral exotic fields are eliminated. In the model derived from the 10D U(8) SYM theory, both of them appeared, and this is an advantage of our model. The remaining massless adjoint fields are called open-string moduli. The presence of them is well known as a notorious problem in string models. Although we might propose some prescription to eliminate them, e.g., orbifold projections [22], we just assume that they vanish somehow in this paper.

Now, we are able to derive the $4 \mathrm{D}$ effective supergravity action, and its spectrum can be calculated exactly. The Kähler metrics and holomorphic Yukawa couplings obtained in this model are almost the same as the 10D U(8) SYM model except for some trivial numerical factors. These were shown in ref. [8,9], and one can calculate the explicit form in accordance with ref. [11]. The phenomenological features of the 10D U(8) SYM model were studied precisely, and a consistent spectrum of the SM particles and their partners was obtained $[8,9]$. However, the gauge kinetic functions are very different. The model proposed in this section leads to nonuniversal gauge kinetic functions, while they were universal in the 10D U(8) SYM model. The gauge kinetic functions of $\mathrm{SU}(3)_{C}$ and $\mathrm{SU}(2)_{L}$ are straightforwardly obtained as

$$
\begin{aligned}
& f_{3}=T_{1}, \\
& f_{2}=S .
\end{aligned}
$$

Before showing that of $\mathrm{U}(1)_{Y}$ gauge group, we should explain breaking of $\mathrm{U}(4)_{C}$ and $\mathrm{U}(2)_{L} \times \mathrm{U}(2)_{R}$. We can introduce the Wilson line parameters to break the Pati-Salam gauge group on the first torus. As the result, $\mathrm{U}(4)_{C}$ is broken down to $\mathrm{U}(3)_{C} \times \mathrm{U}(1)_{C^{\prime}}$, and $\mathrm{U}(2)_{R}$ gauge is also broken to $\mathrm{U}(1)_{R^{\prime}} \times \mathrm{U}(1)_{R^{\prime \prime}}$. Thus, there are five $\mathrm{U}(1)$ gauge symmetries:

$$
\begin{array}{rlrl}
\mathrm{U}(1)_{a} & \equiv \mathrm{U}(1) \subset \mathrm{U}(3)_{C}, & \mathrm{U}(1)_{b} \equiv \mathrm{U}(1)_{C^{\prime}}, & \mathrm{U}(1)_{c} \equiv \mathrm{U}(1) \subset \mathrm{U}(2)_{L}, \\
\mathrm{U}(1)_{d} \equiv \mathrm{U}(1)_{R^{\prime}}, & \mathrm{U}(1)_{e} \equiv \mathrm{U}(1)_{R^{\prime \prime}} . &
\end{array}
$$




\begin{tabular}{|ccccccc|}
\hline Contents & $Q_{a}$ & $Q_{b}$ & $Q_{c}$ & $Q_{d}$ & $Q_{e}$ & $Q_{Y}$ \\
\hline$Q$ & 1 & 0 & -1 & 0 & 0 & $1 / 6$ \\
$L$ & 0 & 1 & -1 & 0 & 0 & $-1 / 2$ \\
$H_{u}$ & 0 & 0 & 1 & -1 & 0 & $1 / 2$ \\
$H_{d}$ & 0 & 0 & 1 & 0 & -1 & $-1 / 2$ \\
$u$ & -1 & 0 & 0 & 1 & 0 & $-2 / 3$ \\
$d$ & -1 & 0 & 0 & 0 & 1 & $1 / 3$ \\
$\nu$ & 0 & -1 & 0 & 1 & 0 & 0 \\
$e$ & 0 & -1 & 0 & 0 & 1 & 1 \\
\hline
\end{tabular}

Table 2. The charges of the five original $\mathrm{U}(1)$ symmetries and $\mathrm{U}(1)_{Y}$ hypercharges defined in eq. (5.3) are shown.

We can define the hypercharge $Q_{Y}$ by a linear combination of these five U(1) symmetries as ${ }^{2}$

$$
Q_{Y}=x Q_{a}+\left(x-\frac{2}{3}\right) Q_{b}+\left(x-\frac{1}{6}\right) Q_{c}+\left(x-\frac{2}{3}\right) Q_{d}+\left(x+\frac{1}{3}\right) Q_{e}
$$

where $x$ is an arbitrary number. The charges for each content are summarized in table 2 . Its gauge coupling constant $g_{Y}$ is then given by

$$
\frac{1}{g_{Y}^{2}}=\frac{x^{2}}{\tilde{g}_{6 \mathrm{D}}^{2}}+\frac{(x-2 / 3)^{2}}{\tilde{g}_{6 \mathrm{D}}^{2}}+\frac{(x-1 / 6)^{2}}{\tilde{g}_{10 \mathrm{D}}^{2}}+\frac{(x-2 / 3)^{2}}{\tilde{g}_{10 \mathrm{D}}^{2}}+\frac{(x+1 / 3)^{2}}{\tilde{g}_{10 \mathrm{D}}^{2}},
$$

where $\tilde{g}_{6 \mathrm{D}}$ and $\tilde{g}_{10 \mathrm{D}}$ are are $4 \mathrm{D}$ effective gauge coupling constants of the $6 \mathrm{D} \mathrm{U}(4)_{C}$ and the $10 \mathrm{D} \mathrm{U}(4)_{L R} \mathrm{SYM}$ theories given by

$$
\tilde{g}_{6 \mathrm{D}}=e^{\langle\phi\rangle / 2} \sqrt{\frac{\alpha^{\prime}}{\mathcal{A}^{(1)}}}, \quad \tilde{g}_{10 \mathrm{D}}=e^{\langle\phi\rangle / 2} \sqrt{\frac{\alpha^{\prime 3}}{\mathcal{A}^{(1)} \mathcal{A}^{(2)} \mathcal{A}^{(3)}}} .
$$

The arbitrary number $x$ used in eq. (5.3) never appear in the hypercharges but it effects only on the gauge coupling, that is, we can control it with this parameter. From eq. (5.4), the $\mathrm{U}(1)_{Y}$ gauge kinetic function reads

$$
f_{1}=\left(2 x^{2}-\frac{4}{3} x+\frac{4}{9}\right) T_{1}+\left(3 x^{2}-\frac{4}{3} x+\frac{7}{12}\right) S,
$$

where the two coefficient given as functions of parameter $x$ should be rational numbers to be consistent with the discrete axionic shift symmetries. We will discuss this constraint again later.

In the rest of this subsection we discuss some typical features of spectrum determined by the Kähler metrics and holomorphic Yukawa couplings. As we have mentioned it is known that a consistent spectrum is obtained with them, but changes of RG flows due to

\footnotetext{
${ }^{2}$ The other four linear combinations are expected to be decoupled from the low-energy effective theory (see section 3.3 of $[8]$ ).
} 
nonuniversal gauge kinetic functions might affect on the spectrum. Moreover, it is nontrivial that the gaugino masses can be varying independently of the other soft parameters at the $M_{\mathrm{GUT}}$ scale.

RG flows of the Yukawa couplings may be deflected compared with the MSSM, with the nonuniversal gauge kinetic functions. Its deflection cannot be drastic because the running of the gauge couplings are hardly changed as will show later. A semi-realistic spectrum of the quarks and leptons obtained in ref. [8] can also be realized in our model.

SUSY spectra of this model are given in the moduli mediation or a combination with the anomaly mediation which is called mirage mediation scenarios [25, 26]. RG effects on the softparameters will be changed in the case of nonuniversal gaugino masses. Although discussions for SUSY spectra in the 10D U(8) model are unable to apply directly to our model, it seems that the deviation will never lead to dangerous tachyons and flavor violations, unless a bizarre boundary condition for squared scalar mass and A-terms are realized in our model. In the following, we remark on possibilities to realize such a boundary condition by the mixed contributions of $S$ and $T_{1}$.

As we will see, SUSY breaking contributions mediated by the moduli fields $S$ and $T_{1}$ must be determined to have a certain ratio in order to obtain desirable nonuniversal gaugino masses. In accordance with ref. [9], dilaton superfield $S$ properly contributes to all the softparameters, and the CMSSM like-spectrum is realized by the sole contribution of $S$. On the contrast, Kähler moduli field $T_{1}$ induces negative contributions to some of squared softmasses, and this cannot dominate SUSY breaking contributions in order to forbid the presence of tachyonic modes. Fortunately, the favored ratio of gaugino masses $\left(M_{2}>M_{3}\right)$ and gauge kinetic functions (5.2) imply that the contribution of dilaton $S$ is much larger than that of Kähler moduli $T_{1}$.

Even when the $T_{1}$ contribution is subdominant, it might lead to unfamiliar boundary conditions of the soft parameters (other than the gaugino masses) at a compactification scale, which has a possibility to spoil the great advantage of non-universal gaugino masses shown in section 2. Although we can estimate the exact spectrum, it is not necessary because it is easy to remove $T_{1}$ SUSY breaking contributions to all the squared scalar masses and A-terms while the moduli still generates the gaugino masses. There are three Kähler moduli fields, $T_{1}, T_{2}$ and $T_{3}$ in our model, and we consider SUSY breaking contributions mediated by them. When they are equal to each other, that is,

$$
\frac{F^{T_{1}}}{t_{1}+\bar{t}_{1}}=\frac{F^{T_{2}}}{t_{2}+\bar{t}_{2}}=\frac{F^{T_{3}}}{t_{3}+\bar{t}_{3}},
$$

where $F^{T_{i}}$ and $t_{i}$ are the VEVs of the auxiliary field and the lowest component of moduli superfield $T_{i}$, their contributions are completely canceled [9]. The modulus $T_{1}$ generates the gaugino masses, while its SUSY breaking contribution is canceled out by the other contributions of $T_{2}$ and $T_{3}$ in the soft scalar masses and the A-terms. Thus, we can control nonuniversal gaugino masses independently of the boundary conditions of the other soft parameters. 


\subsection{Gauge coupling unification and gaugino masses}

This section analyzes the nonuniversal gauge kinetic functions. In studies of nonuniversal gaugino masses [3-5], the gauge coupling constants are set to be unified as is in the MSSM. The exact gauge coupling unification is not essentially important but drastic changes of the gauge couplings may not relax the fine-tunning of the $\mu$-parameter. At least, without the gauge coupling unification, the specific ratios of the gaugino masses (2.1) would not be reliable. To discuss the gauge coupling unification, we define the compactification scale in this model by the mass scale of the lightest KK mode as,

$$
M_{C}=1 / \sqrt{\mathcal{A}^{(1)}} .
$$

The magnetic fluxes and the Wilson lines have the mass scale as high as $M_{C}$. Thus, the gauge groups are broken down at this scale.

The easiest way to realize the gauge coupling unification in our model is that two types of $4 \mathrm{D}$ effective gauge coupling constants, $\tilde{g}_{6}$ and $\tilde{g}_{10}$, are chosen to a unified value which the MSSM predicts at the $M_{\mathrm{GUT}}$ scale, $4 \pi / g_{a}^{2} \sim 24$, and the SUSY breaking and the compactification scales are set to be $10^{3} \mathrm{GeV}$ and $M_{\mathrm{GUT}}$, respectively. (Note that, the SUSY breaking scale here is defined as a typical scale at which the SUSY particles are decoupled from the SM particles.)

In the D-brane picture of type IIB string theory, more constraints will be imposed on these scales and gauge coupling constants. We are studying the SYM theories but it is worth verifying the consistency. In the framework of type IIB string theory, the 4D Planck mass scale and the $4 \mathrm{D}$ effective gauge coupling of Dp-branes are given by (Note that $g_{5}$ and $g_{9}$ given in this expression are equivalent to the $4 \mathrm{D}$ effective couplings of $6 \mathrm{D}$ and $10 \mathrm{D}$ SYM theories, $\tilde{g}_{6}$ and $\tilde{g}_{10}$, respectively.)

$$
\begin{aligned}
M_{\mathrm{pl}} & =\frac{2 e^{-2 \phi}}{(2 \pi)^{7} \alpha^{4}} V, \\
\frac{1}{g_{p}^{2}} & =\frac{e^{-\phi}}{(2 \pi)^{p-2} \alpha^{\prime(p-3) / 2}} V_{p-3},
\end{aligned}
$$

where $e^{\phi}=g_{\mathrm{st}}$ and $\alpha^{\prime}=1 / M_{\mathrm{st}}^{2}$, and $g_{\mathrm{st}}$ and $M_{\mathrm{st}}$ represent a string coupling and a string scale, respectively. $V$ is the volume of $6 \mathrm{D}$ extra compact space, and $V_{p-3}$ denotes the volume of cycles which the Dp-branes wrap. In these expressions, when we set the two gauge couplings as $4 \pi^{2} / g_{5}^{2}=4 \pi^{2} / g_{9}^{2}=24$, taking the realistic value of $4 \mathrm{D}$ Planck mass and the SUSY condition $\mathcal{A}^{(1)} / \mathcal{A}^{(2)}=\mathcal{A}^{(1)} / \mathcal{A}^{(3)}=3$ into account, we find

$$
\begin{aligned}
M_{\mathrm{st}} & \sim 4.31 \times 10^{18} \mathrm{GeV}, \\
M_{C} & \sim 3.96 \times 10^{17} \mathrm{GeV} .
\end{aligned}
$$

The compactification scale is slightly deviated from the $M_{\mathrm{GUT}}$ scale. The gauge couplings evolve to the compactification scale $M_{C}$ through the $M_{\mathrm{GUT}}$ scale within the MSSM RG equations. Although this means that the gauge coupling unification might be missed, the exact form of gauge coupling unification is not required and it is sufficient to at least assert 
that the favored ratios of gaugino masses (2.1) are also reliable in our model. Another estimation of these scales given in the appendix supports this discussion.

We calculate the gaugino masses at the compactification scale. In the framework of supergravity, the gaugino masses are easily calculated by using a formula with the specific forms of gauge kinetic functions (5.2) and (5.5) as follows,

$$
M_{a}=F^{m} \partial_{m} \ln \left(\operatorname{Re} f_{a}\right)+\frac{b_{a} g_{a}^{2}}{16 \pi^{2}} \frac{F^{C}}{C_{0}} .
$$

In the assumption of the gauge coupling unification like the MSSM, the gauge couplings $g_{a}$ and coefficients $b_{a}$ are given as $4 \pi / g_{a}^{2}=24$ and $\left(b_{1}, b_{2}, b_{3}\right)=(33 / 5,1,-3)$, and we find

$$
\begin{aligned}
& M_{3}=\frac{F^{T_{1}}}{t_{1}+\bar{t}_{1}}+\frac{-1}{32 \pi} \frac{F^{C}}{C_{0}}, \\
& M_{2}=\frac{F^{S}}{s+\bar{s}}+\frac{1}{96 \pi} \frac{F^{C}}{C_{0}} \\
& M_{1}=\left(2 x^{2}-\frac{4}{3} x+\frac{4}{9}\right) \frac{F^{T_{1}}}{t_{1}+\bar{t}_{1}}+\left(3 x^{2}-\frac{4}{3} x+\frac{7}{12}\right) \frac{F^{S}}{s+\bar{s}}+\frac{11}{160 \pi} \frac{F^{C}}{C_{0}},
\end{aligned}
$$

where $s$ and $t_{1}$ represents the VEVs of the lowest component fields of the moduli superfields $S$ and $T_{1}$, and $F^{S}$ and $F^{T_{1}}$ are the VEVs of their auxiliary fields. $C_{0}$ and $F^{C}$ are VEVs of the lowest component field and an auxiliary filed of a chiral compensator superfield of the supergravity. The following reparametrization simplifies the above expressions of gaugino masses,

$$
M_{\mathrm{SUSY}} \equiv \frac{F^{T_{1}}}{t_{1}+\bar{t}_{1}}, \quad R^{S} \equiv \frac{1}{M_{\mathrm{SUSY}}} \frac{F^{S}}{s+\bar{s}}, \quad R^{C} \equiv \frac{1}{4 \pi^{2}} \frac{1}{M_{\mathrm{SUSY}}} \frac{F^{C}}{C_{0}} .
$$

The gaugino masses are then expressed by

$$
\begin{aligned}
& M_{3}=M_{\mathrm{SUSY}}\left(1-\frac{\pi}{8} R^{C}\right), \\
& M_{2}=M_{\mathrm{SUSY}}\left(R^{S}+\frac{\pi}{24} R^{C}\right), \\
& M_{1}=M_{\mathrm{SUSY}}\left(\left(2 x^{2}-\frac{4}{3} x+\frac{4}{9}\right)+\left(3 x^{2}-\frac{4}{3} x+\frac{7}{12}\right) R^{S}+\frac{11 \pi}{40} R^{C}\right) .
\end{aligned}
$$

Their ratios which is of our interest are independent of $M_{\mathrm{SUSY}}$. It should be determined to be of $\mathcal{O}(1 \mathrm{TeV})$ being consistent with experimental results.

We determine the value of parameter $x$ in the assumption of gauge coupling unification as follows. The gauge coupling unification $\left\langle\operatorname{Re} f_{a}\right\rangle=6 / \pi$ is realized by $\langle\operatorname{Re} S\rangle=\left\langle\operatorname{Re} T_{1}\right\rangle=$ $6 / \pi$ in (5.2) and (5.5), so we assume that these moduli fields are stabilized satisfying this relation by a stabilization mechanism somehow. In this assumption, we find the following condition on the parameter $x$

$$
\left(2 x^{2}-\frac{4}{3} x+\frac{4}{9}\right)+\left(3 x^{2}-\frac{4}{3} x+\frac{7}{12}\right)=1,
$$

which is equivalent to $x=(8 \pm \sqrt{59}) / 30$. Note that, the value of $x$ should be a rational number for the coefficient of $\mathrm{U}(1)_{Y}$ gauge kinetic functions $\left(2 x^{2}-\frac{4}{3} x+\frac{4}{9}\right.$ and $\left.3 x^{2}-\frac{4}{3} x+\frac{7}{12}\right)$ 


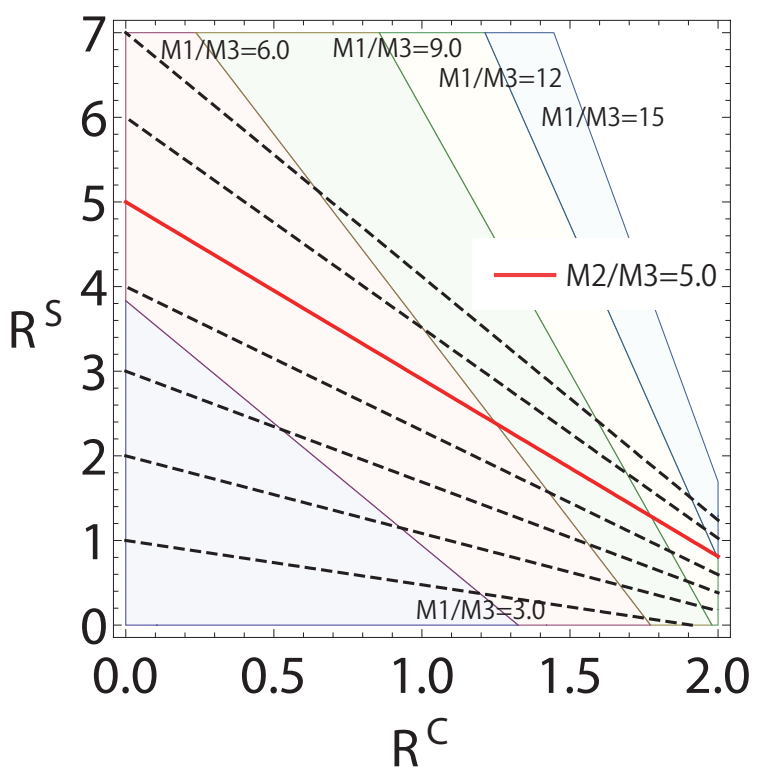

Figure 1. The seven transversal lines represent the ratio of the wino mass to the gluino mass, corresponding to $M_{2} / M_{3}=1,2, \ldots, 7$ from the bottom line. The red solid line, which is one of the seven contours, corresponds to $M_{2} / M_{3}=5.0$. The colored shades show the other ratios, $M_{1} / M_{3}$. We obtain the ratio $0 \leq M_{1} / M_{3} \leq 3$ in the purple region, and $3 \leq M_{1} / M_{3} \leq 6,6 \leq M_{1} / M_{3} \leq 9$, $9 \leq M_{1} / M_{3} \leq 12$ and $12 \leq M_{1} / M_{3} \leq 15$ are obtained in the red, green, yellow and cyan regions, respectively.

to be rational numbers, otherwise the discrete axionic shift symmetries of the moduli fields cannot be explained. However, any rational number cannot satisfy the unification condition (5.10) exactly. This means the complete gauge unification requires slight corrections to the culculations of the gauge couplings, which can originate from $\alpha^{\prime}$-corrections, KK-modes and so on. We have another alternative; the complete unification is not necessary to enjoy the benefit of nonuniversal gaugino masses, but also in this case, the three gauge couplings are almost unified within low-scale SUSY breaking scenarios. Therefore, the irrational number $x=(8 \pm \sqrt{59}) / 30$ must be pretty close to a rational number which is ideal for theoretical consistency. In the following, we adopt $x=(8+\sqrt{59}) / 30$ even though it cannot be accepted since it is sufficient for our purpose to demonstrate the nonuniversarity of gaugino masses.

We estimate the ratios $M_{1} / M_{3}$ and $M_{1} / M_{3}$ on $\left(R_{S}, R_{C}\right)$-plane as shown in figure 1 . In this figure, the mass ratio of the wino and gluino masses are represented by six black dashed lines and a red solid line. These seven contours correspond to $M_{2} / M_{3}=1.0,2.0, \ldots, 7.0$ from the bottom, and the red solid line indicates the most favored one $M_{2} / M_{3}=5.0$. Five colored regions of this plane represent the mass ratio of the bino and the gluino. Each of the purple, red, green, yellow and cyan regions corresponds to $0 \leq M_{1} / M_{3} \leq 3.0$, $3.0 \leq M_{1} / M_{3} \leq 6.0,6.0 \leq M_{1} / M_{3} \leq 9.0,9.0 \leq M_{1} / M_{3} \leq 12.0$ and $12 \leq M_{1} / M_{3} \leq 15$, respectively.

Compared with to the favored mass ratios (2.1), the bound $M_{1} / M_{3} \geq-3$ is always satisfied in all the parameter space, and the other $3.0 \leq M_{2} / M_{3} \leq 5.5$ is also realized 
in the wide region of the parameter space. In particular, the very natural region $5.2 \leq$ $M_{2} / M_{3} \leq 5.5$ can be available. We see that the mechanism to relax the fine-tunning by the nonuniversal gaugino masses will work correctly in our model.

\section{Conclusions and discussions}

We have constructed a model of nonuniversal gaugino masses on the basis of a mixture of $6 \mathrm{D}$ U(4) SYM theory and 10D U(4) SYM theory compactified on three 2D-tori with magnetic fluxes, motivated by stable D-brane systems. In higher-dimensional SYM systems, the toroidal compactifications with magnetic fluxes are able to generate the flavor structure of the SM, and SUSY preserving configurations of magnetic fluxes lead to MSSM-like models. In such models derived from single SYM theories, the gauge kinetic functions of the three SM gauge fields are given by a single modulus universally, ${ }^{3}$ and their couplings have the exactly same form. The gaugino masses must then degenerate at a certain high-energy scale. However, in 4D effective actions derived from two SYM theories defined in different dimensional spacetime, the moduli couplings to the SM gauge fields are involved by two moduli fields, and the three gauge kinetic functions are distinguishable, which generate the non-universal gaugino masses.

Our model leads to nonuniversal gauge kinetic functions containing two moduli fields, where we have studied the gauge coupling constants and the gaugino masses. Since we have found that the unification of gauge coupling constants can be roughly realized as is in the MSSM, the favored ratios of gaugino masses estimated in the MSSM [4] are also being reliable in our model. This is supported by another calculation given in the appendix. The numerical analysis of gaugino masses has shown that our model can yield the gaugino masses with the ideal ratios. Thus, we have proposed a concrete model of nonuniversal gaugino masses in a top-down approach on the basis of a magnetized toroidal compactification of SYM system, where the mechanism to enhance the Higgs boson mass and relax the fine-tunning will work.

The conventional parameter space of the MSSM was certainly well motivated to study primarily. However, we see that the conventional parameter space gives mass spectra mostly accompanied by any of troublesome today in the light of the latest experimental and observational data, such as, the fine-tunning of $\mu$-parameter and lack of candidates for the dark matter. In this situation, unconventional parameter space of the MSSM has been getting attractive. Indeed, nonuniversal gaugino mass is one of them and the attractive prospect has been found. We should then remark on how to realize such an unconventional spectrum desirable from the phenomenological point of view, strictly, how to obtain unconventional boundary conditions for parameters of the MSSM at a certain high scale. A possibility to realize such values of parameter in theories for a high-energy physics should be taken into account more seriously. Such discussion will lead to restrictions on some of unconventional parameter space, or conversely, it is expected that we can infer

\footnotetext{
${ }^{3}$ This is valid in SYM theories, but is nontrivial in string models, such as, D-brane models. Corrected gauge kinetic functions might be able to be calculated with DBI actions and so on in magnetized toroidal compactifications, but it is beyond the scope of this paper.
} 
theories describing a high-energy physics, which we cannot directly reach today, on the basis of the obtained experimental data.

\section{Acknowledgments}

K.S. was supported by a Grant-in-Aid for JSPS Fellows No. 25.4968 from the Ministry of Education, Culture, Sports, Science and Technology in Japan.

\section{A RGE effects on the gauge coupling constants}

This appendix calculates the RG effects on gauge coupling constants in more detail. In our model, the coupling constants of $\mathrm{SU}(3)_{C}$ and $\mathrm{SU}(2)_{L}$ gauge symmetries are given by the 6D and 10D SYM couplings, respectively. A linear combination of the two gauge kinetic functions can be identified with that of the $\mathrm{U}(1) Y$ gauge symmetry. In the combination, there remains the parameter $x$ with which we can control the gauge coupling constant and gaugino mass simultaneously. This should be fixed to satisfy relations between the gauge couplings $\tilde{g}_{6}=g_{3}, \tilde{g}_{10}=g_{2}$ and $g_{1}$. Indeed, we adopted a certain value of $x$ to obtain the gauge coupling unification in section 5. In other words, we can always choose this parameter to approximately realize the experimental value of $\mathrm{U}(1)_{Y}$ coupling constant. Thus, we are allowed to concentrate on the other two gauge coupling constants here.

We solve the RGEs for the gauge coupling constants of $\mathrm{SU}(3)_{C}$ and $\mathrm{SU}(2)_{L}$. The two experimental values evolve in accordance with the SM RGEs from the electroweak scale $\Lambda_{\mathrm{EW}}$ to a typical SUSY breaking scale $\Lambda_{\mathrm{SUSY}}$. From $\Lambda_{\mathrm{SUSY}}$ scale to the compactification scale defined as $M_{C}=1 / \sqrt{\mathcal{A}^{(1)}}$, we can calculate the running of gauge couplings by using the RGEs within the MSSM. Lastly, the obtained values at $M_{C}$ scale will further evolve to the string scale within U(4) YM theories, and these will be consistent with theoretical calculation shown in eq. (5.7). For $\mathrm{SU}(3)_{C}$ gauge, these are summarized as

$$
4 \pi \frac{e^{-\phi}}{(2 \pi)^{3} \alpha^{\prime}} \mathcal{A}^{(1)}=-\frac{b_{5}}{2 \pi}\left(t_{\mathrm{st}}-t_{\mathrm{C}}\right)-\frac{\tilde{b}_{3}}{2 \pi}\left(t_{\mathrm{C}}-t_{\mathrm{SUSY}}\right)-\frac{b_{3}}{2 \pi}\left(t_{\mathrm{SUSY}}-t_{\mathrm{EW}}\right)+\alpha_{3 \exp }^{-1}
$$

where the coefficient of beta functions, $b_{3}$ and $\tilde{b}_{3}$, are given by -7 (SM) and -3 (MSSM). The coefficient $b_{5}=-4$ is related to the $\mathrm{U}(4)_{C}$ SYM theory which contains an adjoint fields and two bifundamental representations. ${ }^{4}$ The experimental value of gauge coupling is defined by $\alpha_{3 \exp }^{-1} \equiv 4 \pi / g_{3 \text {,exp }}^{2}$. Each energy scale $t$ is defined as follows,

$$
\begin{aligned}
t_{\mathrm{st}} & \equiv \log \left(M_{s t} / \Lambda_{0}\right) \\
t_{\mathrm{C}} & \equiv \log \left(M_{C} / \Lambda_{0}\right) \\
t_{\mathrm{SUSY}} & \equiv \log \left(M_{\mathrm{SUSY}} / \Lambda_{0}\right) \\
t_{\mathrm{EW}} & \equiv \log \left(M_{Z} / \Lambda_{0}\right),
\end{aligned}
$$

where $\Lambda_{0}$ is an input scale and $M_{Z}$ represents the mass of $Z$ boson.

\footnotetext{
${ }^{4}$ This calculation does not contain the relevant KK modes. Their contributions would be negligible because the compactification scale and the string scale are almost equal in any cases.
} 
For $\mathrm{SU}(2)_{L}$ coupling constant, the similar relation between, the experimental values of $\mathrm{SU}(2)_{L}$ coupling constant and theoretical representation given at the string scale, is given by

$$
4 \pi \frac{e^{-\phi}}{(2 \pi)^{7} \alpha^{\prime 3}} \mathcal{A}^{(1)} \mathcal{A}^{(2)} \mathcal{A}^{(3)}=-\frac{b_{9}}{2 \pi}\left(t_{\mathrm{st}}-t_{\mathrm{C}}\right)-\frac{\tilde{b}_{2}}{2 \pi}\left(t_{\mathrm{C}}-t_{\mathrm{SUSY}}\right)-\frac{b_{2}}{2 \pi}\left(t_{\mathrm{SUSY}}-t_{\mathrm{EW}}\right)+\alpha_{2 \exp }^{-1},
$$

where the coefficients are given as $b_{9}=4, \tilde{b}_{2}=1$ and $b_{2}=-19 / 64$.

Eqs. (A.1) and (A.2) can be more simplified by using the 4D Planck mass (5.6), the SUSY condition $\mathcal{A}^{(1)} / \mathcal{A}^{(2)}=\mathcal{A}^{(1)} / \mathcal{A}^{(3)}=3$, and typical values of $M_{\mathrm{SUSY}}=10^{3} \mathrm{GeV}$ and $M_{\mathrm{Z}}=10^{2} \mathrm{GeV}$, and then, they will be simultaneous equations for $M_{\mathrm{st}}$ and $M_{C}$. As the result, we find

$$
\begin{aligned}
& M_{\mathrm{st}}=2.78 \times 10^{18} \mathrm{GeV} \\
& M_{\mathrm{C}}=2.65 \times 10^{17} \mathrm{GeV} .
\end{aligned}
$$

This calculation of gauge couplings leads to the almost same result as one obtained in assumption of the rough gauge coupling unification shown in eqs. (5.8) and (5.9). This means that deviation from the MSSM in terms of the running of gauge couplings is small enough for the ideal ratios of gaugino masses (2.1) estimated in the MSSM to be applicable to our model directly.

Open Access. This article is distributed under the terms of the Creative Commons Attribution License (CC-BY 4.0), which permits any use, distribution and reproduction in any medium, provided the original author(s) and source are credited.

\section{References}

[1] ATLAS collaboration, Observation of a new particle in the search for the Standard Model Higgs boson with the ATLAS detector at the LHC, Phys. Lett. B 716 (2013) 1 [arXiv: 1207.7214] [INSPIRE].

[2] CMS collaboration, Observation of a new boson at a mass of $125 \mathrm{GeV}$ with the CMS experiment at the LHC, Phys. Lett. B 716 (2012) 30 [arXiv:1207.7235] [INSPIRE].

[3] H. Abe, T. Kobayashi and Y. Omura, Relaxed fine-tuning in models with non-universal gaugino masses, Phys. Rev. D 76 (2007) 015002 [hep-ph/0703044] [INSPIRE].

[4] H. Abe, J. Kawamura and H. Otsuka, The Higgs boson mass in a natural MSSM with nonuniversal gaugino masses at the GUT scale, Prog. Theor. Exp. Phys. 2013 (2013) 013B02 [arXiv: 1208.5328] [INSPIRE].

[5] S. Antusch, L. Calibbi, V. Maurer, M. Monaco and M. Spinrath, Naturalness of the non-universal MSSM in the light of the recent Higgs results, JHEP 01 (2013) 187 [arXiv: 1207.7236] [INSPIRE].

[6] C. Bachas, A way to break supersymmetry, hep-th/9503030 [INSPIRE].

[7] D. Cremades, L.E. Ibáñez and F. Marchesano, Computing Yukawa couplings from magnetized extra dimensions, JHEP 05 (2004) 079 [hep-th/0404229] [INSPIRE]. 
[8] H. Abe, T. Kobayashi, H. Ohki, A. Oikawa and K. Sumita, Phenomenological aspects of $10 D$ SYM theory with magnetized extra dimensions, Nucl. Phys. B 870 (2013) 30 [arXiv: 1211.4317] [INSPIRE].

[9] H. Abe, J. Kawamura and K. Sumita, The Higgs boson mass and SUSY spectra in $10 D$ SYM theory with magnetized extra dimensions, Nucl. Phys. B 888 (2014) 194 [arXiv:1405.3754] [INSPIRE].

[10] H. Abe, T. Kobayashi, H. Ohki and K. Sumita, Superfield description of $10 D$ SYM theory with magnetized extra dimensions, Nucl. Phys. B 863 (2012) 1 [arXiv:1204.5327] [INSPIRE].

[11] H. Abe, T. Horie and K. Sumita, Superfield description of $(4+2 n)$-dimensional SYM theories and their mixtures on magnetized tori, arXiv:1507.02425 [INSPIRE].

[12] R. Barbieri and G.F. Giudice, Upper bounds on supersymmetric particle masses, Nucl. Phys. B 306 (1988) 63 [INSPIRE].

[13] L. Randall and R. Sundrum, Out of this world supersymmetry breaking, Nucl. Phys. B 557 (1999) 79 [hep-th/9810155] [INSPIRE].

[14] G.F. Giudice, M.A. Luty, H. Murayama and R. Rattazzi, Gaugino mass without singlets, JHEP 12 (1998) 027 [hep-ph/9810442] [INSPIRE].

[15] M. Dine and W. Fischler, A phenomenological model of particle physics based on supersymmetry, Phys. Lett. B 110 (1982) 227 [INSPIRE].

[16] C.R. Nappi and B.A. Ovrut, Supersymmetric extension of the $\mathrm{SU}(3) \times \mathrm{SU}(2) \times \mathrm{U}(1)$ model, Phys. Lett. B 113 (1982) 175 [inSPIRE].

[17] L. Álvarez-Gaumé, M. Claudson and M.B. Wise, Low-energy supersymmetry, Nucl. Phys. B 207 (1982) 96 [INSPIRE].

[18] H. Abe, K.-S. Choi, T. Kobayashi and H. Ohki, Higher order couplings in magnetized brane models, JHEP 06 (2009) 080 [arXiv: 0903.3800] [INSPIRE].

[19] N. Marcus, A. Sagnotti and W. Siegel, Ten-dimensional supersymmetric Yang-Mills theory in terms of four-dimensional superfields, Nucl. Phys. B 224 (1983) 159 [INSPIRE].

[20] N. Arkani-Hamed, T. Gregoire and J.G. Wacker, Higher dimensional supersymmetry in $4 D$ superspace, JHEP 03 (2002) 055 [hep-th/0101233] [INSPIRE].

[21] L.E. Ibáñez and A.M. Uranga, String theory and particle physics: an introduction to string phenomenology, Cambridge Univ. Pr., Cambridge U.K. (2012).

[22] H. Abe, T. Kobayashi and H. Ohki, Magnetized orbifold models, JHEP 09 (2008) 043 [arXiv: 0806.4748] [INSPIRE].

[23] H. Abe, K.-S. Choi, T. Kobayashi and H. Ohki, Three generation magnetized orbifold models, Nucl. Phys. B 814 (2009) 265 [arXiv:0812.3534] [INSPIRE].

[24] T.-H. Abe et al., Classification of three-generation models on magnetized orbifolds, Nucl. Phys. B 894 (2015) 374 [arXiv: 1501.02787] [INSPIRE].

[25] K. Choi, K.S. Jeong and K.-I. Okumura, Phenomenology of mixed modulus-anomaly mediation in fluxed string compactifications and brane models, JHEP 09 (2005) 039 [hep-ph/0504037] [INSPIRE].

[26] M. Endo, M. Yamaguchi and K. Yoshioka, A bottom-up approach to moduli dynamics in heavy gravitino scenario: superpotential, soft terms and sparticle mass spectrum, Phys. Rev. D 72 (2005) 015004 [hep-ph/0504036] [INSPIRE]. 